BRIEF COMMUNICATION

\title{
'Purpose in Life' as a psychosocial resource in healthy aging: an examination of cortisol baseline levels and response to the Trier Social Stress Test
}

\author{
Nia Fogelman ${ }^{1}$ and Turhan Canli ${ }^{1,2,3,4}$
}

'Purpose in Life' (Purpose) is associated with healthy aging, but it is unknown whether this association is causal. Conceptualizing Purpose as a form of psychosocial resource, one mechanism promoting health could be the regulation of stress hormones. To test this hypothesis, we recruited 44 older community-dwelling adults to examine the association between Purpose and cortisol at baseline, in response to, and recovery from, an acute social laboratory stressor. Purpose did not predict cortisol baseline or reactivity, but did predict a faster recovery to pre-stress baseline levels. The health benefits of Purpose in aging may therefore reflect the combination of a normal stress response, which serves an adaptive benefit of allostasis, with an accelerated stress recovery, which reduces the burden of allostatic load. This model should be tested in future studies using larger samples, multiple related constructs, and longitudinal designs that include participants' health records.

npj Aging and Mechanisms of Disease (2015) 1, 15006; doi:10.1038/npjamd.2015.6; published online 28 September 2015

'Purpose in Life' (Purpose), a sense of meaning and goal directedness, may serve as a psychosocial resource to promote healthy aging. A high sense of Purpose is associated with reduced mortality, ${ }^{1}$ Alzheimer's disease ${ }^{2}$ and myocardial infarction. ${ }^{3}$

One mechanism by which Purpose may promote healthy aging is by regulation of stress hormones, balancing the adaptive benefit of allostasis against the cost of allostatic load. ${ }^{4}$ We addressed this possibility using the Trier Social Stress Test (TSST) ${ }^{5}$ in older community-dwelling adults. We predicted that Purpose predicts cortisol levels at baseline, in response to, or recovery following TSST exposure.

Forty-four participants were recruited by flyers and advertisements from the area surrounding Stony Brook University. The sample was $55-90$ years $(M=65.4$, s.d. $=8.3 ; 15$ males), and included Caucasian (90.9\%), Hispanic (4.5\%), Asian (2.3\%) and African-American (2.3\%) individuals. Participants were informed that the study was on stress and aging, and included saliva samples, a blood draw, psychological questionnaires and an interview-like task. Self-reported exclusion criteria were: psychiatric illness, diabetes, psychoactive drug medication, substance or alcohol abuse, smoking, hormonal medication and current immense stress. The Committee on Research Involving Human Subjects of Stony Brook University, Stony Brook, USA, approved this study.

The TSST followed a well-established protocol, ${ }^{5}$ involving a public speaking and math task in front of two unresponsive judges. Saliva was collected using Salivettes (Sarstedt, Germany) at arrival, 2 min prior to the TSST, and at 2, 10, 20, 30, 45, 60, 90 and $105 \mathrm{~min}$ post stress task. Salivary cortisol concentrations were measured in a contract laboratory (Rohleder, Brandeis University). Purpose was assessed using a 10 -item questionnaire ${ }^{1}$ to measure meaning and goal-oriented feelings about the future. A 12-item version of the Trier Inventory of Chronic Stress (TICS) assessed how often participants subjectively experienced facets of chronic stress over the past 3 months.

Data analyses were conducted using SPSS v. 22 (Armonk, NY, USA). Baseline cortisol levels were log transformed to correct positive skew. Reactivity was measured as (1) area under the curve with respect to increase ${ }^{6}$ and (2) as peak-baseline. ${ }^{7}$ Cortisol recovery was the percentage difference between values at peak and $45 \mathrm{~min}$ post stressor: ((cortisol $45 \mathrm{~min}$ post stressor-peak cortisol)/peak cortisol $\times 100$ ). Any data point more than 2 s.d. from the mean was winsorized. ${ }^{8}$ Multiple regression analyses tested the association between Purpose and each cortisol measure, controlling for age, gender and time of day ('morning': 0900-1100 h; 'afternoon': 1100-1500 h; or 'evening': 1500-1730 h).

The regression model for cortisol baseline was significant $(P<0.001)$, but this was driven by participants' arrival time. The peak reactivity models were not significant. The model for cortisol recovery was significant $\left(P=0.02 ; R^{2}=0.288\right)$, and this was driven by Purpose $(\beta=-0.386, P=0.008)$. Corresponding scatterplots are shown in Figure 1, and Table 1 lists all regression models.

We conducted a bivariate correlation between Purpose and TICS scores (representing allostatic load). This revealed that the two measures were significantly inversely related $(r=-0.46, \quad P=0.002)$. A regression model in which TICS scores were substituted for Purpose scores was not significant $(P=0.085)$.

Purpose predicted faster cortisol recovery after a social stressor, consistent with a study reporting faster stress recovery after exposure to negative images. ${ }^{9}$ We found Purpose did not predict cortisol baseline levels or TSST reactivity. A TSST study ${ }^{7}$ that included Purpose as one element of a composite variable also found an association with cortisol reactivity but not with baseline

\footnotetext{
${ }^{1}$ Department of Psychology, Integrative Neuroscience, Stony Brook University, Stony Brook, NY, USA; ${ }^{2}$ Department of Radiology, Stony Brook University, Stony Brook, NY, USA; ${ }^{3}$ Program in Neuroscience, Stony Brook University, Stony Brook, NY, USA and ${ }^{4}$ Program in Genetics, Stony Brook University, Stony Brook, NY, USA.

Correspondence: T Canli (turhan.canli@stonybrook.edu)

Received 29 April 2015; revised 29 June 2015; accepted 13 July 2015
} 


Purpose, cortisol and the TSST
N Fogelman and T Canli
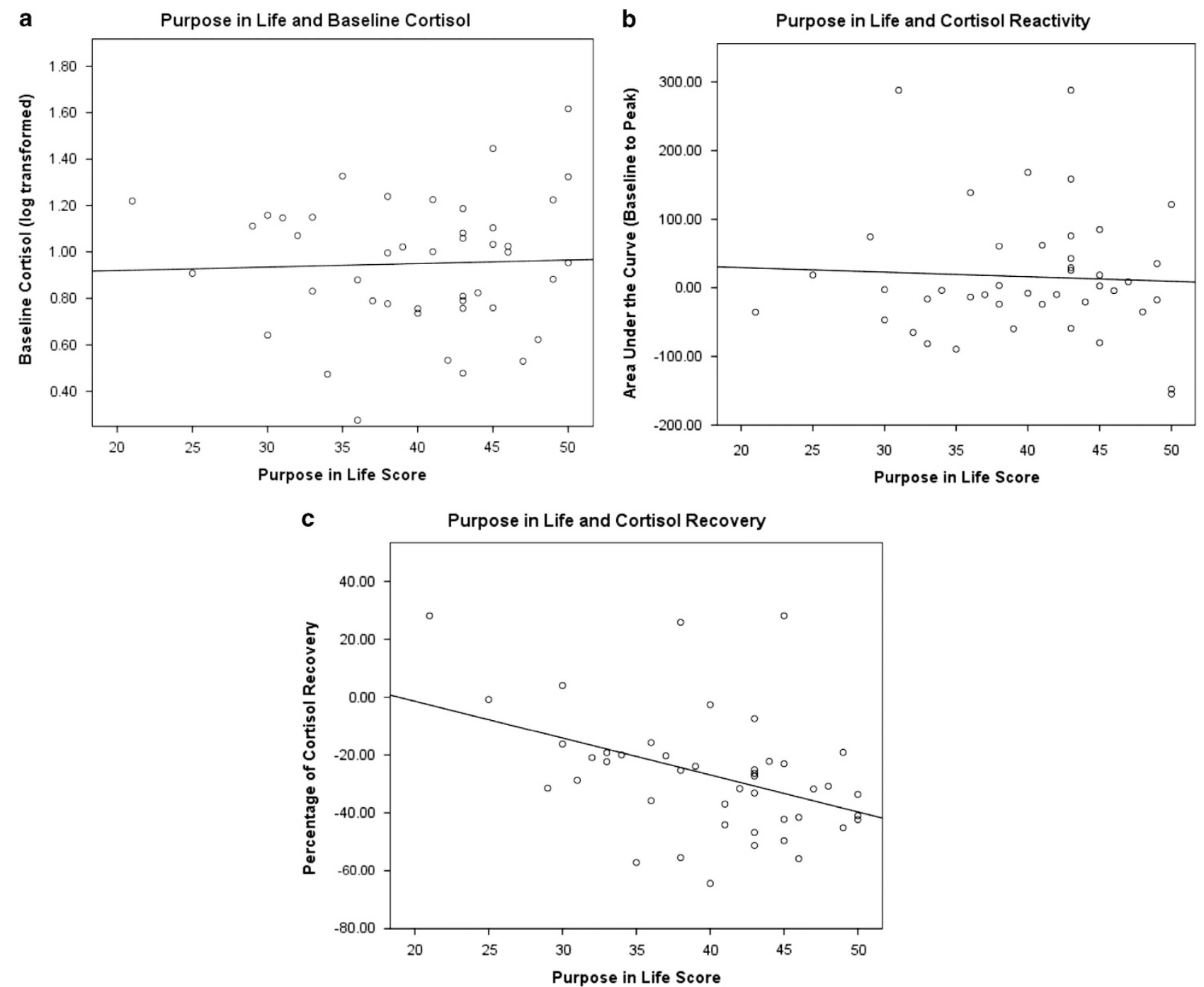

Figure 1. Cortisol baseline, peak and recovery as a function of 'Purpose in Life'. The graphs above depict the relationship between Purpose in Life and baseline cortisol (a), cortisol reactivity (b) and cortisol recovery (c). Purpose in Life only significantly related to cortisol recovery.

Table 1. Model values for baseline, reactivity and recovery

\begin{tabular}{|c|c|c|c|c|c|c|c|c|}
\hline & \multicolumn{2}{|c|}{ Baseline } & \multicolumn{2}{|c|}{ Reactivity (AUCi) } & \multicolumn{2}{|c|}{$\begin{array}{c}\text { Reactivity } \\
\text { (peak-baseline) }\end{array}$} & \multicolumn{2}{|c|}{ Recovery } \\
\hline & $\beta$ & P-value & $\beta$ & P-value & $\beta$ & P-value & $\beta$ & P-value \\
\hline Time 1 & -0.351 & 0.344 & -0.125 & 0.465 & -0.071 & 0.689 & -0.201 & 0.219 \\
\hline Time 2 & -0.657 & $<0.001$ & 0.242 & 0.167 & 0.258 & 0.158 & -0.218 & 0.190 \\
\hline Age & -0.190 & 0.139 & -0.330 & 0.036 & -0.258 & 0.108 & 0.156 & 0.282 \\
\hline
\end{tabular}

Abbreviations: AUCi, area under the curve with respect to increase; Purpose, Purpose in Life.

Time 1 represents the afternoon group compared with the morning group. Time 2 represents the evening group compared with the morning group. 
cortisol or recovery. These findings contrast ours, perhaps due to differences in the age of study cohorts, measurement of cortisol recovery, or the use of a composite score combining Purpose with other constructs.

Our study has three limitations as follows: limited sample size to address related constructs, lack of objective health status, and cross-sectional rather than longitudinal design. In future research, we plan to examine overlapping constructs such as well-being, which conceptualizes Purpose as a subscale. ${ }^{10}$ In addition, although we did obtain subjective information leading us to believe that our sample was physically and mentally healthy, medical records may provide a more objective assessment. Finally, a longitudinal study would allow us to measure how Purpose may affect cortisol recovery throughout the aging process.

Purpose may facilitate stress reappraisal as we found that it was inversely correlated with the subjective experience of chronic stress (proxy for allostatic load). High subjective chronic stress on its own did not predict cortisol recovery. Therefore, our data suggest that individuals with high Purpose show adaptive stress reactivity (allostasis), but reduce chronic stress via cortisol recovery (allostatic load). This response style, accumulating over a lifetime of daily social stressors could be a powerful mechanism promoting healthy aging.

\section{ACKNOWLEDGEMENTS}

We thank the many former members of the Canli Lab, postdocs, graduate students and undergraduate research assistants for their help in running the experimental sessions and data entry. This work was funded by the National Institute on Aging (National Institutes of Health) 1 R01 AG034578-01 awarded to TC.

\section{CONTRIBUTIONS}

NF analyzed the data and wrote the first draft of the manuscript. TC supervised the design and execution of the study and wrote the manuscript. Both authors read and approved the final manuscript.

\section{COMPETING INTERESTS}

The authors declare no conflict of interest.

\section{REFERENCES}

1 Boyle PA, Barnes LL, Buchman AS, Bennett DA. Purpose in life is associated with mortality among community-dwelling older persons. Psychosom Med 2009; 71: 574.

2 Boyle PA, Buchman AS, Barnes LL, Bennett DA. Effect of a purpose in life on risk of incident Alzheimer disease and mild cognitive impairment in communitydwelling older persons. Arch Gen Psychiatry 2010; 67: 304-310.

3 Kim ES, Sun JK, Park N, Kubzansky LD, Peterson C. Purpose in life and reduced risk of myocardial infarction among older US adults with coronary heart disease: a two-year follow-up. J Behav Med 2013; 36: 124-133.

4 McEwen BS. Protective and damaging effects of stress mediators. N Engl J Med 1998; 338: 171-179.

5 Kirschbaum C, Pirke KM, Hellhammer DH. The 'Trier Social Stress Test'-a tool for investigating psychobiological stress responses in a laboratory setting. Neuropsychobiology 1993; 28: 76-81.

6 Pruessner JC, Kirschbaum C, Meinlschmid G, Hellhammer DH. Two formulas for computation of the area under the curve represent measures of total hormone concentration versus time-dependent change. Psychoneuroendocrinology 2003; 28: 916-931.

7 Taylor SE, Burklund $\sqcup$, Eisenberger NI, Lehman BJ, Hilmert CJ, Lieberman MD. Neural bases of moderation of cortisol stress responses by psychosocial resources. J Pers Soc Psychol 2008; 95: 197.

8 Ghosh D, Vogt A. Outliers: an evaluation of methodologies. Joint Statistical Meetings 2012, p 3455-3460.

9 Schaefer SM, Boylan JM, van Reekum CM, Lapate RC, Norris CJ, Ryff CD et al. Purpose in life predicts better emotional recovery from negative stimuli. PLOS ONE 2013; 8: e80329.

10 Ryff CD. Happiness is everything, or is it? Explorations on the meaning of psychological well-being. J Pers Soc Psychol 1989; 57: 1069.

(c) (i) This work is licensed under a Creative Commons Attribution 4.0 International License. The images or other third party material in this article are included in the article's Creative Commons license, unless indicated otherwise in the credit line; if the material is not included under the Creative Commons license, users will need to obtain permission from the license holder to reproduce the material. To view a copy of this license, visit http://creativecommons.org/licenses/ by/4.0/ 